\title{
An intersectionality lens is needed to establish a global view of equity, diversity and inclusion
}

\author{
Rassim Khelifa ${ }^{1}$ and Hayat Mahdjoub ${ }^{2}$ \\ ${ }^{1}$ The University of British Columbia \\ ${ }^{2}$ Universitat Zurich
}

October 19, 2021

\begin{abstract}
Equity, diversity, and inclusion (EDI) have become essential considerations in different academic fields in recent years, attracting an increasing number of voices and perspectives from different groups. While recent contributions have shed light on the barriers faced by some groups, the concept of EDI and implementation of solutions are still in their infancy in ecology and evolution. There is a clear lack of an intersectionality framework that is more inclusive of the global diversity of researchers. As researchers in ecology and evolution from the Global South and Global North with different backgrounds, we recognize the need to present a global view of EDI in order to highlight the role of intersectionality where researchers from Global South are not only impeded by discrimination, but also by other cultural, linguistic, and socioeconomic factors that affect their level of training, ultimately reducing their likelihood of reaching leadership positions. We present a simple model of intersectionality that explains the main drivers of the variation in academic success among researchers, and highlight that most of the variation is determined by factors that individuals have no control over (e.g. place of birth, gender, ethnicity). We recommend measures to increase the representation of the global diversity in the field of ecology and evolution in order to collectively solve global societal and environmental issues.
\end{abstract}

An intersectionality lens is needed to establish a global view of equity, diversity and inclusion Rassim Khelifa ${ }^{1,2}$ \& Hayat Mahdjoub ${ }^{2}$

${ }^{1}$ Institute for Resources, Environment and Sustainability, University of British Columbia, Vancouver, 4292202 Main Mall. Vancouver, BC Canada V6T 1Z4

${ }^{3}$ Laboratory of Biology, Water and Environment, Faculty SNV-STU, University of 8 May 1945 Guelma, Guelma 24000, Algeria

${ }^{4}$ Faculty of Forestry, University of British Columbia, 2424 Main Mall Vancouver, BC Canada V6T 1Z4

Corresponding author:

rassimkhelifa@gmail.com

Word count: 1998

Running head: Intersectionality in academia

Keywords: Global South, Discrimination, bias, developing countries, Science, Ecology, Evolution

Data Accessibility Statement: No new data are presented.

Author Contribution: RK and HM conceived the idea, analyzed the data, and wrote the manuscript.

\section{Abstract}


Equity, diversity, and inclusion (EDI) have become essential considerations in different academic fields in recent years, attracting an increasing number of voices and perspectives from different groups. There is a clear lack of an intersectionality framework that is more inclusive of the global diversity of researchers. As researchers from the Global South, we recognize the need to present a global view of EDI in order to highlight the role of intersectionality between discrimination and other cultural, linguistic, and socioeconomic factors that ultimately reduce the likelihood of researchers from Global South of reaching leadership positions. We present a simple model of intersectionality that explains the main drivers of the variation in academic success among researchers, and highlight that these drivers are often correlated. We recommend measures to increase the representation of the global diversity of researchers in order to collectively solve global societal and environmental issues.

\section{Main}

There has been a growing interest in the barriers that prevent scientists with different backgrounds from succeeding in science (Maas et al. 2021). The recognition of the importance of equity, diversity, and inclusion (EDI) has sparked the interest of scientists in academia in recent years (Tseng et al. 2020), especially since 2020. Recent events had a large impact in the academic world, including in the field of Ecology and Evolution. In response, scientists and institutions questioned some of the fundamental aspects of the system in an attempt to identify the occurrence of systemic discrimination that affects students and researchers from minority groups. There is no longer any uncertainty whether barriers exist for some groups inhibiting their ability to excel and obtain leadership positions in the workforce. This is observable in the simple fact that these groups are vastly underrepresented in student body, faculty positions, authorship on articles, and on editorial boards of most scientific journals (Nuñez et al.2019). While actionable changes are required to make academia more equitable and inclusive (Urbina-Blancoet al. 2020), many fundamental elements of EDI are still not well defined, and the current concepts and vision are unfortunately relatively narrow and localized.

The need for intersectionality in EDI

Intersectionality is a multifaceted framework for understanding the impact of the cumulative occurrence of privileges or barriers on individual success (Hearn \& Louvrier 2015). It is one way to decipher the 'real' drivers of academic opportunities and success among researchers worldwide by looking at major intrinsic and extrinsic factors that affect individual ability to survive and reach leadership positions in the global scientific scene. We propose a simplified formula of intersectionality that explains academic success (S) as a function of four components: training $(\mathrm{T})$, language $(\mathrm{L})$, Networking $(\mathrm{N})$, and discrimination $(\mathrm{D})[\mathrm{S}=f$ (T,L,N,D)] (Fig. 1a), where each component is determined by an array of factors.

Training is a metric that depends on educational institutions, the economy of the country, and one's mentors. Universities play a crucial role in providing the resources (infrastructure), expertise (hiring the best scientists), funding and other opportunities that affect the quality of education of students, their willingness to pursue a science career, and the scientific productivity of researchers. The economy of a country is also a large determinant of the quality of training because rich countries can invest more money into education and research, providing a superior pedagogical and academic environment (Das et al.2013). Also, the choice of an academic supervisor shapes the quality of training of students, the originality and impact of the research questions they ask, the size of the academic networks they build, and the reputation of the journals in which they publish (Clement et al. 2020).

Language is the second major component of scientific success, particularly because English is the lingua franca in science and the ability of individuals to access the content of the literature depends on their knowledge of English (Ramírez-Castañeda 2020). There is a tradeoff in using a universal language. The advantage is unification and facilitation of science communication, but the disadvantage is that the singular use of the English language limits access to science to those who are proficient in English (Nuñezet al. 2021), which does not include those living in many less developed regions of the world. English proficiency intersects and interacts with the quality of training. For countries whose main language is not English, they need to have a good infrastructure and teach students English from an early age to have that quality of training (Amano 
et al. 2021).

Networking is a powerful component of academic success that facilitates not only collaborations but also the transfer of knowledge and the discovery of new research, techniques and tools. Even in this internet era, physical meetings are still the best way to interact with people and create real bonds. Researchers who often go to conferences overseas have more opportunities to meet international leading researchers in their field and create ties with them. However, there is a high difference in the ease of traveling overseas among nationalities where those from the Global South often need to apply for visa, pay fees, and go through heavy paper work to travel to conferences. Together with the lack of funding for conferences, the possibilities of international networking for Global South researchers are very limited.

Discrimination is another force that prevents people from succeeding and reaching leadership positions (Chaudhury \& Colla 2021). Studies have recently highlighted institutional discrimination against black, indigenous and people of color (BIPOC), women, LGBTQIA + , and people with disabilities (e.g. Hofstraet al. 2020). These biases are often both visual and non-visual, that is, based on implicit biases such as inferring individual performance based on the name and affiliation of people (Pinholster 2016). Discrimination is thus a critical point of scientific success as, regardless of the level of training and language proficiency that individuals have, discrimination could drastically reduce one's probability of success.

Depending on the place of birth, socioeconomic status, health, gender, and sexual orientation, researchers could be affected by a single to multiple barriers at once that not only reduce their likelihood of succeeding in academia but also exacerbate the amount of effort and time to spend to reach the expectations of leading scientists. Intersectionality shows that addressing EDI from a single dimension (T, L, N or D) is not effective to depict the cumulative barriers that individuals encounter, and a multivariate analysis is needed to address equity (Fig. 1b).

\section{Correlation between the drivers of academic success}

The high international disparity in academic success, particularly between the Global North and Global South, is mainly due to the accumulation of differences in $T, L$, and $N$ (Fig. 2b). Unsurprisingly, there is a positive correlation between $T, L$, and $N$ (Fig. 2c) where researchers in wealthy countries often have better English proficiency and much less restrictions to travel worldwide than researchers in poor countries. This positive association generates an academic system where researchers "either have it all or have nothing". Therefore, a global intersectionality framework of the four drivers of academic success reveals that researchers from the Global South are limited by $T, L, N$ and $D$, but those from the Global North are mostly affected by $D$ (Fig. 2d). Hence, to succeed in academia, researchers from unprivileged backgrounds must overcompensate by spending extra efforts and money learning English at a late age, seeking independent training, finding access to journals and books, carrying out research with limited to no funding and adequate infrastructure, and moving to countries in the Global North with cumbersome difficulties. The realization that most determinants of $T, L, N$ and Dare beyond the control of individual students and scientists is the first step towards an exhaustive comprehension of EDI.

Geographic scale acknowledgment in EDI

Scientists are human and so they are more prone to focus on local factors that affect the academic success of individuals in their working environment. The reason why most of the discussion on EDI from the Global North is about $D$ is that local variations in $T, L$, and $N$ are not large enough to spark a debate. When we discuss EDI from a global perspective, say Global North vs. Global South, T, $L$, and $N$ become the main drivers of variation in academic success. Therefore, we should realize that $D$ occurs everywhere in the world (local dominant groups are typically advantaged), whereas disadvantages in $T, L$, and $N$ are localized (some countries struggle more than others). True inclusivity should not be partial and EDI discussion should integrate a geographic scale when invoking 'diversity' to highlight whether the discussion is about local or global diversity.

Here, we distinguish between the microdiversity - people living in the same place, sharing the same scientific 
infrastructure, having different genders, skin colors, sexual orientations, disabilities, etc., and macrodiversity - the sum of microdiversities across the globe, including a plethora of institutions and people from different countries, ethnicity, and backgrounds (Fig. 2c). Having such stratification of diversity acknowledges the 'existence' of a huge diversity of people outside our working environment that we do not see and interact with but share the same objectives and go through immense barriers to reach them.

Implications of intersectionality in global challenges

There are many global challenges that require the involvement of many nationalities from the Global South and the Global North as well as the empowerment of local populations worldwide. For instance, the goal of global biodiversity conservation is very difficult to achieve because expertise is currently localized, mostly unilingual, and not equally transferred across countries (Amano \& Sutherland 2013). Countries that meet the highest number of barriers to excel in science often have global biodiversity hotspots where research are expertise are most urgently needed. However, the most biodiverse areas of the world still receive the least conservation research, often conducted by scientists from western countries (Wilson et al. 2016), a practice commonly known as helicopter research. Addressing these issues should involve diversifying expertise and ameliorating our ways to transfer knowledge to countries that are currently not leaders in science, which involve solving issues related to $T, L, N$, and $D$. Until then, addressing global challenges such as climate change, sustainable development, human population growth, water quality and security, and gender equality, is difficult.

\section{Conclusion and perspectives}

Our intersectionality analysis of academic success (Fig. 1a) helps build a framework to determine how the scientific community, funders and institutions should tackle the issues that impede EDI, in particular, and science, in general. Here, we discuss solutions for tackling the barriers at the microdiversity and macrodiversity scale.

Microdiversity. Promoting microdiversity in institutions and societies needs measures that prevent discrimination in the academic environment and increase the participation of minority groups in research. The absence of role models for minority groups in research institutions, editorial boards, and funding organizations is a major problem in the current system. The lack of training in EDI and intersectionality in most universities is also an issue that should be solved with proper education to provide the appropriate support to students and researchers from visible minorities. Other effective strategies might involve (1) welcoming a diverse research group by encouraging students from ethnic and religious minorities and different sexual orientations to join research groups; (2) creating support programs for minorities to guide upcoming foreign students and help them to adapt and feel included in the academic environment; (3) establishing regular EDI discussion groups to educate students and faculty members of the department about the discrimination and unconscious biases; (4) inviting speakers from marginalized groups in order to highlight their scientific contributions; (5) encouraging reading and citing research from visible and non-visible minorities, and (6) working on identifying problems of implicit biases.

Macrodiversity. The idea that we should only tackle discrimination to increase the representation of researchers from the Global South is inadequate as it is only one part of this issue. It is crucial to also improve access to science for researchers from the GS. This requires finding solutions to the language barrier, knowledge transfer, and scientific networking. Decreasing the burden of the language barrier by creating linguistic peer-review platforms where preprints from researchers with English as Foreign Language are reviewed by English proficient volunteers such as graduates and senior researchers is a promising avenue. There is a need to generalize the multilingual abstract and title in all journals to help scientists with limited English proficiency to access the scientific content of research articles. Also, promoting international collaborations of researchers from the Global North with researchers and students from the Global South by encouraging co-authorship and co-supervision as well as organizing major international conferences in countries of the Global South will help the exchange of ideas and perspectives and compensate for the lack of local expertise in developing countries. To overcome the problem of visa restrictions for traveling abroad, a problem 
that researchers from the Global South often face, international conferences should integrate virtual remote attendance to increase the diversity of nationalities and include everyone in the discussion of major issues.

\section{Acknowledgments}

R.K. was supported by the Swiss National Science Foundation (P400PB_191139). We thank Leithen K. M'Gonigle, Melissa Guzman, Amy Angert and Darwin Sodhi for helpful comments and suggestions. R.K. is grateful to the Equity, Diversity, and inclusion discussion group at UBC for fruitful discussions.

\section{Conflict of interest}

The author declares no conflict of interest.

\section{References}

Amano, T., Rios Rojas, C., Boum Ii, Y., Calvo, M. \& Misra, B.B. (2021). Ten tips for overcoming language barriers in science. Nature Human Behaviour .

Amano, T. \& Sutherland, W.J. (2013). Four barriers to the global understanding of biodiversity conservation: wealth, language, geographical location and security. Proc. R. Soc. London, Ser. B , 280, 20122649.

Chaudhury, A. \& Colla, S. (2021). Next steps in dismantling discrimination: Lessons from ecology and conservation science. Conserv. Lett. , 14, e12774.

Clement, L., Leung, K.N., Lewis, J.B. \& Saul, N.M. (2020). The Supervisory Role of Life Science Research Faculty: The Missing Link to Diversifying the Academic Workforce?J. Microbiol. Biol. Educ. , 21.

Das, J., Do, Q.-T., Shaines, K. \& Srikant, S. (2013). US and them: The geography of academic research.Journal of Development Economics , 105, 112-130.

Hearn, J. \& Louvrier, J. (2015). Theories of difference, diversity, and intersectionality. In: The Oxford handbook of diversity in organizations . Oxford University Press, p. 62.

Hofstra, B., Kulkarni, V.V., Galvez, S.M.-N., He, B., Jurafsky, D. \& McFarland, D.A. (2020). The diversityinnovation paradox in science. Proc. Natl. Acad. Sci. U.S.A., 117, 9284-9291.

Maas, B., Pakeman, R.J., Godet, L., Smith, L., Devictor, V. \& Primack, R. (2021). Women and Global South strikingly underrepresented among top-publishing ecologists. Conserv. Lett. , e12797.

Nunez, M.A., Barlow, J., Cadotte, M., Lucas, K., Newton, E., Pettorelli, N. et al. (2019). Assessing the uneven global distribution of readership, submissions and publications in applied ecology: obvious problems without obvious solutions. J. Appl. Ecol. , 56, 4-9.

Nunez, M.A., Chiuffo, M.C., Pauchard, A. \& Zenni, R.D. (2021). Making ecology really global. Trends Ecol. Evol. , 36, 766-769.

Pinholster, G. (2016). Journals and funders confront implicit bias in peer review. Science, 352, 1067-1068.

Ramirez-Castaneda, V. (2020). Disadvantages in preparing and publishing scientific papers caused by the dominance of the English language in science: The case of Colombian researchers in biological sciences. PloS one, 15, e0238372.

Tseng, M., El-Sabaawi, R.W., Kantar, M.B., Pantel, J.H., Srivastava, D.S. \& Ware, J.L. (2020). Strategies and support for Black, Indigenous, and people of colour in ecology and evolutionary biology. Nat. Ecol. Evol. , 4, 1288-1290.

Urbina-Blanco, C.A., Jilani, S.Z., Speight, I.R., Bojdys, M.J., Friščić, T., Stoddart, J.F. et al.(2020). A diverse view of science to catalyse change: valuing diversity leads to scientific excellence, the progress of science and, most importantly, it is simply the right thing to do. We must value diversity not only in words, but also in actions. NRC Research Press 1840 Woodward Drive, Suite 1, Ottawa, ON K2C 0P7. 
Wilson, K.A., Auerbach, N.A., Sam, K., Magini, A.G., Moss, A.S.L., Langhans, S.D. et al. (2016). Conservation research is not happening where it is most needed.PLoS Biol. , 14, e1002413.

\section{Figure captions}

Fig. 1. A simplified diagram of how intersectionality affects academic success, highlighting the barriers and solutions. (a) Academic success depends on training $(T)$, language $(L)$, networking $(N)$, and discrimination $(D)$. Here, $T$ and $D$ are affected by several factors. $T$ depends on the institution, economy of the country, and supervisor. Discrimination is mainly driven by whether an individual belongs to BIPOC (black, indigenous, or people of color), particular gender, LGBTQUIA + , has disabilities, and others (non-visual implicit biases based on name or institution). (b) The consideration of one dimension obscures the real variation among individuals. For instance, individuals with the same linguistic skills might have received different training in different countries, or individuals with the same training can be affected differently by discrimination.

Fig. 2. Intersectionality in academia across four axes: socioeconomy, language, networking, and discrimination. A. Maps showing the gross domestic product per capita of 2018 (GDP) (a), English fluency index $(\mathrm{EF})(\mathrm{b})$, and visa-free score (number of countries that a country is allowed to travel to without a visa) (c) in 96 English as Foreign language countries. All three variables were log-transformed. B. Correlation between GDP, EF, and the visa-free score, highlighting three Global South countries (Nigeria, Tunisia, and Cameroon) and three Global North countries (Germany, Belgium, and Spain) (d-f). The black lines are linear regressions. Theoretical chart applying the equation of academic success [Success $=f$ (Training $[T]$, Language $[L]$, Networking $[N]$, Discrimination $[D])]$ in random individuals from the six countries (g). The chart shows that because economic status is generally positively correlated with English proficiency and networking opportunities, researchers from the Global South have often at least three components $(T, L$, and $N)$ below average (-) while researchers from the Global North have typically these three components above average $(+)$. The chart also illustrates the difference between microdiversity and macrodiversity, highlighting that within each country, researchers from different backgrounds (ethnicity or gender) could be affected by discrimination even if they are located in the Global North. Data on EF were obtained from (https://www.ef.com/ca/epi/). Data on GDP were obtained from WorldBank (https://data.worldbank.org/). Data on visa-free scores were obtained from Henley \& Partners (https://www.henleyglobal.com/passport-index). 

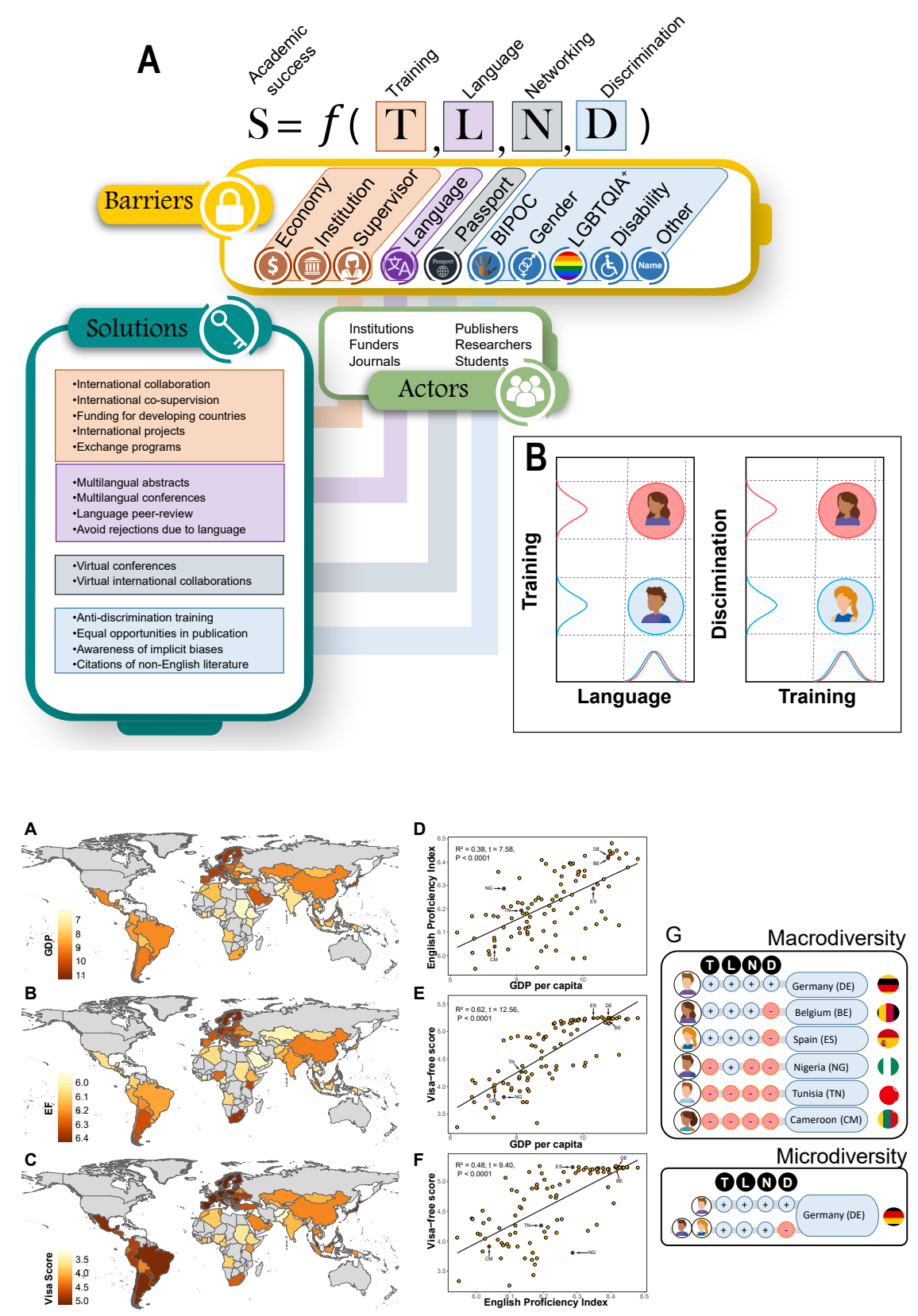